\title{
Elevated levels of circulating IL-18BP and perturbed regulation of IL-18 in schizophrenia
}

\author{
Ilaria Palladino ${ }^{1}$, Francesca Salani ${ }^{1}$, Antonio Ciaramella ${ }^{1}$, Ivo Alex Rubino ${ }^{2}$, Carlo Caltagirone ${ }^{1,2}$, Sabrina Fagioli ${ }^{1}$, \\ Gianfranco Spalletta ${ }^{1}$ and Paola Bossù ${ }^{1,3^{*}}$
}

\begin{abstract}
Background: The pleiotropic pro-inflammatory cytokine Interleukin (IL)-18 has been proposed to play a role in schizophrenia, since elevated circulating levels of its protein and altered frequencies of genetic variants in its molecular system are reported in schizophrenic patients.

Methods: We analyzed 77 patients with schizophrenia diagnosis (SCZ) and 77 healthy control subjects (HC) for serum concentration of both IL-18 and its natural inhibitor, the IL-18 binding protein (IL-18BP).

Results: We confirmed that serum levels of total IL-18 are significantly increased in SCZ, as compared to HC. However, due to a highly significant increase in levels of circulating IL-18BP in SCZ, as compared to HC, the levels of free, bioactive IL-18 are not significantly different between the two groups. In addition, the relationships between the levels of IL-18 and its inhibitor, as well as between the two molecules and age appear dissimilar for SCZ and HC. In particular, the elevated levels of IL-18BP, likely a consequence of the body's attempt to counteract the early prominent inflammation which characterizes schizophrenia, are maintained in earlier and later stages of the disease. However, the IL-18BP elevation appears ineffective to balance the IL-18 system in younger SCZ patients, while in older patients the levels of circulating bioactive IL-18 are comparable to those of HC, if not lower.

Conclusions: In conclusion, these findings indicate that the IL-18 system is perturbed in schizophrenia, supporting the idea that this pro-inflammatory cytokine might be part of a pathway of genetic and environmental components for vulnerability to the disease.
\end{abstract}

Keywords: Interleukin-18, Interleukin-18 Binding Protein, Inflammation, Schizophrenia

\section{Background}

Interleukin (IL)-18 is a pleiotropic pro-inflammatory molecule acting as a potent promoter of chronic inflammation, autoimmunity and age-dependent inflammatory processes [1,2]. Recently, IL-18 has been found to play a diverse array of functions in non-immune tissues as the central nervous system [3], and its abnormal expression has been observed both centrally and peripherally in neuropsychiatric disorders such as Alzheimer's disease and stroke $[4,5]$.

\footnotetext{
* Correspondence: p.bossu@hsantalucia.it

'Clinical and Behavioral Neurology, IRCCS Fondazione Santa Lucia, Via Ardeatina 306, 00179 Rome, Italy

${ }^{3}$ Department of Clinical and Behavioral Neurology, Experimental Neuro-psychobiology Lab, IRCCS Santa Lucia Foundation, Via Ardeatina, 306 I-00179 Rome, Italy

Full list of author information is available at the end of the article
}

The bioactivity of IL-18 is modulated by a secreted binding protein (IL-18BP) with neutralizing abilities, which binds IL-18 with high affinity $\left(K_{\mathrm{d}}=0.4 \mathrm{nM}\right)$ and prevents its interaction with the receptor [6,7]. IL-18BP is constitutively present in the serum of healthy humans at molar excess when compared with IL-18 [8], and it appears to be modulated during the life. In fact, since IL18 participates in fundamental inflammatory processes that increase during the aging process [2] and IL-18BP serum levels tend to increase in old age with evidence of strong increment in healthy centenarians [9], it has been suggested that IL-18BP retains a remarkable protective role by acting to limit the impact of age-related inflammation. In addition, IL-18BP appears to be strongly modulated in pathological circumstances, as it increases with the increase of IL-18 production in inflammatory conditions [10], likely operating a negative feedback mechanism

\section{Biomed Central}


that is able to reduce IL-18-elicited immune responses [11].

Schizophrenia is a familiar multigenic disorder with probable neurodevelopmental origins, characterized by a wide range of psychotic symptoms that appear in adolescence and persist throughout the life of affected individuals. Among the several possible hypotheses formulated to explain the genesis of schizophrenia, immune system vulnerability is one of the most recognized $[12,13]$. A possible link between immune function changes and schizophrenia development is the stimulation of cytokine response. This has prompted renewed interest in cytokines as markers of immune imbalance in schizophrenia and cytokine-centered hypotheses of schizophrenia pathogenesis have been recently proposed [14]. In agreement with the idea that imbalances of pro- and anti-inflammatory cytokines may contribute to the onset of psychotic symptoms and the progressive loss of brain tissue in schizophrenia, IL-18 has been proposed to be connected to this disorder. In particular, five polymorphisms in genes related to the IL-18 pathway, including IL-18 receptor genes, have been associated with schizophrenia [15] and some variants of the $I L-18$ gene have been recently related to the development of schizophrenia symptoms [16]. Furthermore, levels of IL-18 circulating protein are higher in affected patients than in controls $[17,18]$ and are associated with the psychopathology of schizophrenia [19].

However, the IL-18 biology in schizophrenia is still unclear and no data are available on the relationship between IL-18 and IL-18BP in affected individuals. Therefore, in the present study we analyzed IL-18 and IL-18BP peripheral levels in subjects with schizophrenia diagnosis (SCZ) in comparison to healthy control subjects $(\mathrm{HC})$

\section{Materials and methods Subjects}

Seventy-seven SCZ diagnosed according to the Diagnostic and Statistical Manual of Mental Disorders-IV edition (DSM-IV) as previously reported [20] were recruited from outpatient clinics in Central Italy. Seventy-seven healthy control subjects $(\mathrm{HC})$ were recruited in the same geographic area and matched with the SCZ for age and gender, as summarized in Table 1. Exclusion criteria were: 1) treatment with anti-inflammatory or immunosuppressive medication; 2) overt infectious disease or auto-immune disease; 3) history of alcohol or drug dependence or traumatic head injury; 4) any past or present major medical or neurological illness. This research was carried out with the ethical approval of Santa Lucia Foundation institutional review board. Written informed consent was obtained from each subject after giving subjects a complete description of the study.

All patients were receiving stable oral doses of one or more atypical antipsychotic drugs such as risperidone,
Table 1 Clinical and biological characteristics of schizophrenic and healthy control subjects

\begin{tabular}{|c|c|c|}
\hline Characteristics & $\begin{array}{l}\text { Healthy control } \\
\text { subjects } \\
(H C, n=77)\end{array}$ & $\begin{array}{l}\text { Schizophrenic } \\
\text { patients } \\
(S C Z, n=77)\end{array}$ \\
\hline Males/Females (n) & $49 / 28$ & $49 / 28$ \\
\hline Age (years \pm SEM) & $41.8 \pm 1.4$ & $40.9 \pm 1.3^{a}$ \\
\hline Serum IL-18 (pg/ml \pm SEM) & $382 \pm 16.5$ & $518.2 \pm 22.1^{b}$ \\
\hline Serum IL-18BP (ng/ml \pm SEM) & $4.9 \pm 0.2$ & $9.8 \pm 0.4^{b}$ \\
\hline Serum free IL-18 (pg/ml \pm SEM) & $239.3 \pm 9.8$ & $253.6 \pm 12.1^{a}$ \\
\hline Disease onset age (years \pm SEM) & - & $25.4 \pm 1.1$ \\
\hline Duration of illness (years \pm SEM) & - & $14.9 \pm 1.6$ \\
\hline $\begin{array}{l}\text { Olanzapine equivalents } \\
\text { (mg/day } \pm \text { SEM })\end{array}$ & - & $22.3 \pm 5.5$ \\
\hline
\end{tabular}

${ }^{a}$ difference is not significant versus $H C ;{ }^{b} P<0.0001$ versus $H C$

quetiapine, and olanzapine. Antipsychotic dosages were converted to estimated equivalent dosages of olanzapine.

\section{Serum and IL-18/IL-18BP measurement}

Serum samples were obtained from all subjects by centrifugation of clotted blood, and aliquots were stored at $-80^{\circ} \mathrm{C}$ until cytokine assays were performed.

Total IL-18, corresponding to the total amount of free IL-18 and IL-18 bound to IL-18BP, was determined by enzyme-linked immunosorbent assay (ELISA), using antibodies unable to distinguish between the free and the bound form of IL-18. More in detail, coating antibody (clone 125-2 H), detecting antibody (clone 159-12B) and standard human recombinant IL-18, were used (MBL, Nagoya, Japan). IL-18BP was measured using commercial ELISA (R\&D Systems Minneapolis, MN, USA) specific for IL-18BPa, the prevalent isoform in humans, in accordance with the manufacturer's instructions. The limit of detection for both assays was $12.5 \mathrm{pg} / \mathrm{mL}$. Concentrations of free IL-18, namely unbound to IL-18BP, resulted from calculation based on the law of mass action, considering 1:1 stoichiometry in the complex of IL-18 and IL-18BP and a dissociation constant $(\mathrm{K} d)$ of $0.4 \mathrm{nM}$, as elsewhere reported in more detail $[10,21]$.

\section{Statistical analysis}

In order to detect statistically significant differences, comparisons between continuous variables were made using the Student's $t$ test. Pearson's correlation coefficient was used to assess the relationships between continuous variables. The level of statistical significance was defined as $P<0.05$. GraphPad (Prism Version 4, San Diego, CA) software was used for statistical analyses.

\section{Results}

Serum levels of IL-18/IL-18BP

The concentration of both IL-18 and IL-18BP was evaluated for the serum of each subject. In order to define the 
levels of bioactive cytokine, free IL-18 was also calculated on the basis of IL-18 and IL-18BP results. Mean values of total or unbound IL-18 and its inhibitor concentration are reported in Table 1, while individual values are reported in the upper panels of Figure 1. Total IL-18 levels were significantly higher in SCZ than HC $(P<0.0001)$. Similarly, serum levels of IL-18BP were significantly higher in SCZ than $\mathrm{HC}(P<0.0001)$. Interestingly, serum levels of free, bioactive IL-18 were not significantly different between SCZ and HC. The levels of the three elements of IL-18 system were not significantly different between males and females and did not correlate significantly with illness duration and antipsychotic drug dosages.

\section{Relationship between IL-18, IL-18BP and age}

In order to evaluate a possible relationship between total IL-18 and IL-18BP levels, we first assessed the relationship existing between the two immune mediators both in SCZ and HC. A positive significant correlation was found in SCZ $(r=0.245, P=0.03)$ but not in HC.

Afterwards, given that IL-18 might participate in agedependent inflammation, we analyzed the direct relationship between the serum concentration of total IL-18, IL-18BP, free IL-18 and age in all subjects. Indeed, a significant positive correlation between serum total IL-18 levels and age was found in $\mathrm{HC}(r=0.268 ; P=0.018)$, but not in SCZ. No significant correlations were observed between the levels of IL-18BP and age in both HC and
SCZ. Differently, an unexpected and highly significant inverse correlation was found between the serum amount of free IL-18 and age in SCZ $(r=-0.432 ; P<0.0001)$ but not in $\mathrm{HC}$.

In order to further investigate the age dependence of the IL-18 system, we compared the changes in the levels of IL-18-related molecules in $\mathrm{HC}$ and SCZ after dividing each main group into two subgroups formed by the youngest ( $\leq 30$ years; $\mathrm{HC}, \mathrm{n}=15$ and $\mathrm{SCZ}, \mathrm{n}=18$ ) and the oldest ( $\geq 50$ years; $\mathrm{HC}, \mathrm{n}=22$ and $\mathrm{SCZ}, \mathrm{n}=19$ ) subjects. As shown in the lower panels of Figure 1, SCZ showed an increase of total IL-18, as compared to $\mathrm{HC}$, in the youngest $(1.82$-fold increase; $P<0.0001)$ but not in the oldest individuals. The levels of IL-18BP were significantly higher in SCZ, as compared to $\mathrm{HC}$, both in the youngest and the oldest subjects, although the increase was slightly higher in the youngest $(2.2$-fold increase; $P<0.0001)$ than the oldest $(1.96$-fold increase; $P<0.0001)$ subgroup. In contrast, the levels of free IL-18 were significantly higher in SCZ than $\mathrm{HC}$ as for the subgroup of the youngest subjects (1.48-fold increase; $P=0.0006)$, while the biologically active cytokine has a tendency to decrease in SCZ versus $\mathrm{HC}$ in individuals with an age $\geq 50$ years (1.24-fold decrease; $P=0.068)$.

\section{Discussion}

It is known that the pro-inflammatory cytokine IL-18 is present in the circulation partly as an inactive complex

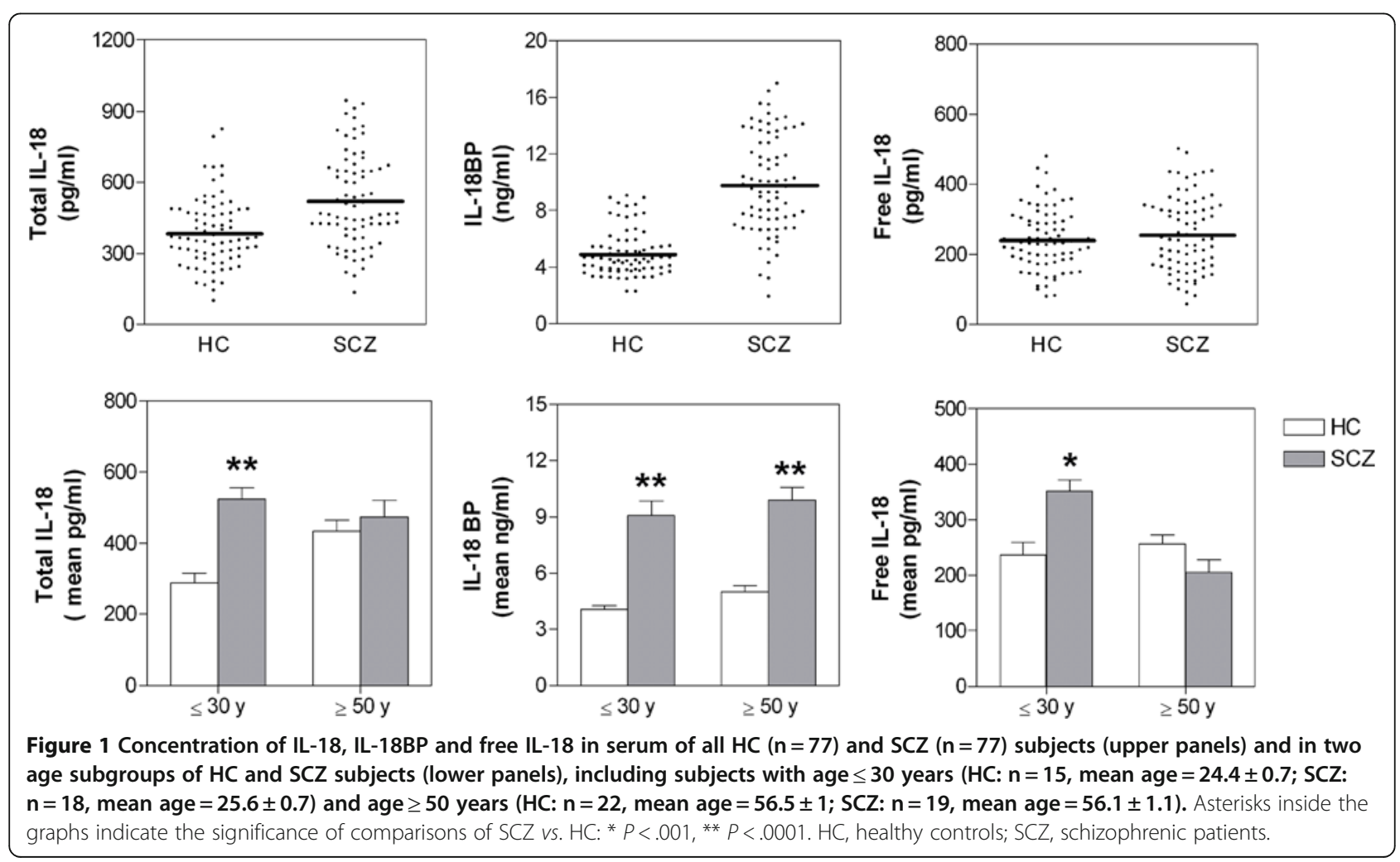


with IL-18BP and partly as free, bioactive IL-18 [6,7]. Previous studies indicate that schizophrenia is paralleled by elevated levels of serum IL-18 without any characterization of the active form of the cytokine $[17,18]$. In the present report we assessed the levels of both IL-18 and IL-18BP in SCZ and age- and gendermatched HC. We confirmed that the total amount of IL18 , including the unbound and the IL-18BP-bound form of the cytokine, is higher in SCZ than in HC. Nevertheless, the main finding of this study is that the cytokine inhibitor IL-18BP is significantly increased in the serum of SCZ, as compared to HC. Interestingly, this elevation is about $40 \%$ higher than that of total IL-18. Thus, the evident increase of IL-18BP in SCZ creates a balance in the levels of free active IL-18, which, in fact, appear comparable between patients and $\mathrm{HC}$ subjects.

As a further indication of the altered modulation of IL18 system in schizophrenia, we found a positive correlation between total IL-18 and its inhibitor only in SCZ and not in HC. This correlation may be linked to the fact that SCZ show, in comparison with $\mathrm{HC}$, a greater range of values in IL-18BP levels. This, together with the observation occurring in autoimmune conditions that IL-18BP is triggered when the IL-18 response is amplified [10], leads us to hypothesize that IL-18BP elevation in SCZ is linked to an early and disease-dependent increase of IL18. Accordingly, when we compared the levels of IL-18 system molecules of SCZ and $\mathrm{HC}$ belonging to different age groups, we confirmed the previous assumption that in $\mathrm{HC}$ there is a trend of IL-18 to increase with age $[2,9]$. Conversely, this age-associated modulation of the IL-18 system appears altered in SCZ, thus strengthening the concept of an IL-18 system perturbation associated with the disorder. We established that in SCZ subjects, as compared to age-matched $\mathrm{HC}$, the increase of total and free IL-18 was observable only in the youngest individuals, where the elevation of IL-18BP did not seem to reach adequate levels to reduce free IL-18 to HC levels, thus reflecting an imbalance which might favor a general inflammatory state in the early phase of the disorder. Noteworthy, in agreement with the condition we have described for young SCZ patients, a number of studies has previously reported disease-related high levels of free IL-18, despite the concomitant over expression of IL$18 \mathrm{BP}[10,22,23]$, which is in accordance with the nature of the interaction between IL-18 and IL-18BP [24].

At variance with young individuals, SCZ subjects with an age $\geq 50$ years did not show levels of total IL-18 significantly different from $\mathrm{HC}$, likely a result of the agedependent increase of total IL-18 values occurring only in $\mathrm{HC}$, which would progressively bring the cytokine levels of the latter to almost reach those of SCZ. Furthermore, in older SCZ the highly significant elevation of IL-18BP persisted and free IL-18 levels appeared slightly lower than those of HC. In this view, a possible modulation of IL-18 system might be envisaged according to the following scenario. In young SCZ, total and free IL-18 increase over normal levels, similar to what has been observed for other pro-inflammatory cytokines, perhaps as a consequence of an inflammatory challenge at the maternal-fetal interface [14]. Therefore, IL-18BP elevation might be interpreted as an effort of the organism to compensate the exaggerated inflammatory response linked to the disorder. This counteraction might become effective in balancing the IL-18-mediated proinflammatory reaction only in the later stages.

In agreement with our findings, other recent studies suggest that SCZ subjects are not characterized by a simple pro-inflammatory profile, but more likely the illness is accompanied by both pro- and anti-inflammatory activated forces, which may involve both monocyte and T-cell networks, probably as a product of an inflammatory system control $[25,26]$. Yet, the sense of this perturbation in IL-18 system, characterized by elevated levels of IL-18BP here described for the first time in the context of schizophrenia, should be deciphered on a time-dependent basis, in the light of its ability to limit harmful inflammation on the one hand and to contribute to increased susceptibility to infection and immunerelated diseases, on the other hand [27]. The early increase in free IL-18, which fades away with age in SCZ, consistently points to the importance of considering age and duration of the illness when discussing the immunerelated pathophysiological mechanisms of schizophrenia. Longitudinal studies that address the kinetic of IL-18 system players in relation to disease progression can further clarify the role of free IL-18 in SCZ patients.

The interpretation of our results should take into consideration some limitations of the present study. We used a naturalistic approach where all SCZ were receiving psychotropic drugs, though antipsychotic medication might be a confounding factor in the analysis of the immune system in schizophrenia. Nonetheless, in this study there was not a relationship between antipsychotic treatment dosages and the cytokine parameters, suggesting that in our conditions treatment and serum IL-18 system changes might be independent. This is consistent with the findings of two other recent studies which report that the antipsychotic treatment does not significantly modulate the levels of IL-18 in the serum of schizophrenic patients $[18,19]$, even though the cytokine produced by patients' blood cells is actually increased by therapy [18]. In conclusion, regardless of whether the IL-18 dysregulation is of genetic, infectious or other origin, our findings strongly suggest that the elevation of IL-18BP found in our SCZ, as well as the whole perturbation of the IL-18 system, might be indeed a specific phenomenon of schizophrenia. Given the hypothesis of immune dysfunction in this illness and 
the emergent interest in inflammatory mechanisms as potential targets for its treatment $[27,28]$, our results may offer new insights into the field. In fact, even though further clarification of IL-18 role in the disease is certainly needed, the described data suggest that more attention should be paid to the analysis of circulating cytokines, which ought to include the contextual measurement of cytokine natural inhibitors and antagonists in order to develop novel and potentially effective anti-inflammatory therapeutic intervention against schizophrenia.

\section{Abbreviations}

DSM-IV: Diagnostic and Statistical Manual of Mental Disorders;

ELISA: Enzyme-Linked Immunosorbent Assay; HC: healthy controls;

IL: interleukin; IL-18BP: interleukin-18 binding protein; SCZ: schizophrenia.

\section{Competing interests}

The authors declare that they have no competing interests.

\section{Authors' contribution}

PB, GS and CC designed and coordinated the study. IAR, SF and GS selected patients and performed all clinical evaluations. IP, FS and AC prepared samples and performed cytokine experiments. All authors made substantial contribution to analysis and discussion of the data. IP wrote the initial draft of the manuscript and PB revised it. All authors made contributions in writing and discussing the manuscript. All authors have read and approved its final version.

\section{Acknowledgements}

This study received financial support from Italian Ministry of Health, Grant RF07-08 and RC-05-06-07-08-09-10-11 (PB and GS). This study was supported by the ERANET-NEURON 2010 grant.

\section{Author details}

${ }^{1}$ Clinical and Behavioral Neurology, IRCCS Fondazione Santa Lucia, Via Ardeatina 306, 00179 Rome, Italy. '2Department of Neuroscience, University Tor Vergata, Via Montpellier 1, 00133 Rome, Italy. ${ }^{3}$ Department of Clinical and Behavioral Neurology, Experimental Neuro-psychobiology Lab, IRCCS Santa Lucia Foundation, Via Ardeatina, 306, I-00179 Rome, Italy.

Received: 6 July 2012 Accepted: 13 August 2012

Published: 22 August 2012

\section{References}

1. Dinarello CA: Interleukin-18, a proinflammatory cytokine. Eur Cytokine Netw 2000, 11:483-486.

2. Dinarello CA: Interleukin 1 and interleukin 18 as mediators of inflammation and the aging process. Am J Clin Nutr 2006, 83:447S-455S.

3. Alboni S, Cervia D, Sugama S, Conti B: Interleukin 18 in the CNS. J Neuroinflammation 2010, 7:9.

4. Bossù P, Salani F, Cacciari C, Picchetto L, Cao M, Bizzoni F, Rasura M, Caltagirone C, Robinson RG, Orzi F, Spalletta G: Disease outcome, alexithymia and depression are differently associated with serum IL-18 levels in acute stroke. Curr Neurovasc Res 2009, 6:163-170.

5. Bossù $P$, Ciaramella $A$, Salani $F$, Vanni $D$, Palladino I, Caltagirone $C$ Scapigliati G: Interleukin-18, from neuroinflammation to Alzheimer's disease. Curr Pharm Des 2010, 16:4213-4224

6. Novick D, Kim SH, Fantuzzi G, Reznikov LL, Dinarello CA, Rubinstein M: Interleukin-18 binding protein: a novel modulator of the Th1 cytokine response. Immunity 1999, 10:127-136.

7. Sims JE: IL-1 and IL-18 receptors, and their extended family. Curr Opin Immunol 2002, 14:117-122.

8. Novick D, Schwartsburd B, Pinkus R, Suissa D, Belzer I, Sthoeger Z, Keane WF, Chvatchko Y, Kim SH, Fantuzzi G, Dinarello CA, Rubinstein M: A novel IL-18BP ELISA shows elevated serum IL-18BP in sepsis and extensive decrease of free IL-18. Cytokine 2001, 14:334-342.

9. Gangemi S, Basile G, Merendino RA, Minciullo PL, Novick D, Rubinstein M, Dinarello CA, Lo Balbo C, Franceschi C, Basili S, D’ Urbano E, Daví G, Nicita-Mauro V, Romano M: Increased circulating Interleukin-18 levels in centenarians with no signs of vascular disease: another paradox of longevity? Exp Gerontol 2003, 38:669-672.

10. Novick D, Elbirt D, Miller G, Dinarello CA, Rubinstein M, Sthoeger ZM: High circulating levels of free interleukin-18 in patients with active SLE in the presence of elevated levels of interleukin-18 binding protein. J Autoimmun 2010, 34:121-126.

11. Mühl H, Kämpfer $H$, Bosmann M, Frank S, Radeke H, Pfeilschifter J: Interferon-gamma mediates gene expression of IL-18 binding protein in nonleukocytic cells. Biochem Biophys Res Commun 2000, 267:960-963.

12. Kinney DK, Hintz K, Shearer EM, Barch DH, Riffin C, Whitley K, Butler R: A unifying hypothesis of schizophrenia: abnormal immune system development may help explain roles of prenatal hazards, post-pubertal onset, stress, genes, climate, infections, and brain dysfunction. Med Hypotheses 2010, 74:555-563.

13. Meyer U: Developmental neuroinflammation and schizophrenia. Prog Neuropsychopharmacol Biol Psychiatry 2011, PMID: 22122877.

14. Watanabe $Y$, Someya $T$, Nawa H: Cytokine hypothesis of schizophrenia pathogenesis: evidence from human studies and animal models. Psychiatry Clin Neurosci 2010, 64:217-230.

15. Shirts BH, Wood J, Yolken RH, Nimgaonkar VL: Comprehensive Evaluation of Positional Candidates in the IL-18 Pathway Reveals Suggestive Associations With Schizophrenia and Herpes Virus Seropositivity. Am J Med Genet B Neuropsychiatr Genet 2008, 147:343-350.

16. Liu J, Liu J, Zhou Y, Li S, Li Y, Song X, Wang J, Wang L, Ying B: Association between promoter variants of interleukin-18 and schizophrenia in a Han Chinese population. DNA Cell Biol 2011, 30:913-917.

17. Tanaka KF, Shintani F, Fujii Y, Yagi G, Asai M: Serum interleukin-18 levels are elevated in schizophrenia. Psychiatry Res 2000, 96:75-80.

18. Reale M, Patruno A, De Lutiis MA, Pesce M, Felaco M, Di Giannantonio M, Di Nicola M, Grilli A: Dysregulation of chemo-cytokine production in schizophrenic patients versus healthy controls. BMC Neurosci 2011, 25:12-13.

19. Xiu MH, Chen DC, Wang D, Zhang K, Dong A, Tang W, Zhang F, Liu L, Liu JH, Liu HB, Yang FD, Kosten TR, Zhang XY: Elevated interleukin-18 serum levels in chronic schizophrenia: association with psychopathology. J Psychiatr Res 2012, 46:1093-1098.

20. Spoletini I, Cherubini A, Banfi G, Rubino IA, Peran P, Caltagirone C, Spalletta G: Hippocampi, thalami, and accumbens microstructural damage in schizophrenia: a volumetry, diffusivity, and neuropsychological study. Schizophr Bull 2011, 37:118-130.

21. Migliorini P, Anzilotti C, Pratesi F, Quattroni P, Bargagna M, Dinarello CA, Boraschi D: Serum and urinary levels of IL-18 and its inhibitor IL-18BP in systemic lupus erythematosus. Eur Cytokine Netw 2010, 21:264-271.

22. Mazodier K, Marin V, Novick D, Farnarier C, Robitail S, Schleinitz N, Veit V, Paul P, Rubinstein M, Dinarello CA, Harlé JR, Kaplanski G: Severe imbalance of IL-18/IL-18BP in patients with secondary hemophagocytic syndrome. Blood 2005, 106:3483-3489.

23. Ludwiczek O, Kaser A, Novick D, Dinarello CA, Rubinstein M, Tilg H: Elevated systemic levels of free interleukin-18 (IL-18) in patients with Crohn's disease. Eur Cytokine Netw 2005, 16:27-33.

24. Kim SH, Eisenstein M, Reznikov L, Fantuzzi G, Novick D, Rubinstein M, Dinarello CA: Structural requirements of six naturally occurring isoforms of the IL-18 binding protein to inhibit IL-18. Proc Natl Acad Sci USA 2000, 97:1190-1195.

25. Drexhage RC, Hoogenboezem TA, Cohen D, Versnel MA, Nolen WA, van Beveren NJ, Drexhage HA: An activated set point of T-cell and monocyte inflammatory networks in recent-onset schizophrenia patients involves both pro- and anti-inflammatory forces. Int $\mathrm{J}$ Neuropsychopharmacol 2011, 14:746-755.

26. Miller BJ, Buckley P, Seabolt W, Mellor A, Kirkpatrick B: Meta-analysis of cytokine alterations in schizophrenia: clinical status and antipsychotic effects. Biol Psychiatry 2011, 70:663-671.

27. Meyer U: Anti-inflammatory signaling in schizophrenia. Brain Behav Immun 2011, 25:1507-1518.

28. Meyer U, Schwarz MJ, Müller N: Inflammatory processes in schizophrenia: a promising neuroimmunological target for the treatment of negative/ cognitive symptoms and beyond. Pharmacol Ther 2011, 132:96-110.

doi:10.1186/1742-2094-9-206

Cite this article as: Palladino et al:: Elevated levels of circulating IL-18BP and perturbed regulation of IL-18 in schizophrenia. Journal of Neuroinflammation 2012 9:206. 